\title{
PROPUESTA DE MATERIAL DIDÁCTICO DESDE EL DISEÑO VISUAL ACOTADO EN LA INCLUSIÓN Y EL ENTORNO EDUCATIVO INFANTIL AUTISTA DEL CENTRO NEUROKIDS
}

\section{Entorno Culura}

Maria Mercedes Ruiz Toledo

mariaruiz@unimayor.edu.co

Institución Universitaria Colegio Mayor Del Cauca

Grupo Rutas - Semillero Engrama

Popayán, Cauca.

Autismo infantil,

diseño visual,

Palabras Clave: inclusión, material didáctico, pedagogía,

psicología. 


\section{Resumen}

a mejora en la educación visual y gráfica de los niños y niñas de 6 a 10 años con Trastorno

- del Espectro Autista (TEA), unida al aprendizaje lúdico, son los dos temas que impulsan el proyecto, con la elaboración de un material didáctico que logra mejorar tanto la enseñanza como su aprendizaje, en el Centro de Rehabilitación Infantil Neurokids, con el fin de suplir la necesidad principal, la cual es la ausencia de material didáctico; por esto a los niños y niñas con este trastorno, se les dificulta el aprendizaje de los temas educativos. Se desarrolló el design thinking como proceso metodológico, pues éste ayuda a generar una solución más precisa a la necesidad y deseo de la población objetivo, ya que permite recolectar información sobre el grupo poblacional, con la finalidad de tener una participación activa e información completa y precisa. Como principal resultado, se obtuvo la mejora tanto de la enseñanza como del aprendizaje, gracias al prototipo -material didáctico- que se elaboró, además de mejorar la comunicación y motivación por aprender temas como: geografía, ortografía, abecedario y matemáticas, al tener en cuenta los elementos visuales y la teoría de inteligencias múltiples formulada por Howard Gardner (1983). Es importante destacar, que el manejo de materiales didácticos físicos, es relevante en el proceso educativo de niños y niñas con autismo, puesto que ayuda a disminuir su aislamiento y aumenta su motivación por aprender.

\section{Introducción}

Al entrar en el mundo del diseño inclusivo, se deben tener en cuenta sus diversas herramientas para proporcionar calidad de vida a un tipo de población, en este caso, para niños y niñas con Trastorno del Espectro Autista (TEA), pues el diseño también es un tema social, debido a que permite un progreso al producir servicios y productos para aportar a diferentes mejoras.

Así entonces, la influencia que tiene lo visual dentro del aprendizaje de los niños y niñas con TEA, se ha estudiado en las disciplinas de psicología y neuropsicología, para asegurar que lo visual tiene gran relevancia en el desarrollo educativo de dichos infantes (Howard Gardner, 1983).

Respecto a este tema, Riviére (2001) afirma:

Desde los años sesenta, setenta y ochenta la educación se ha convertido en el tratamiento principal del autismo, teniendo como uno de los factores principales, la creación de centros educativos dedicados específicamente al autismo, llevando consigo también el manejo de materiales educativos en este tipo de centros.

Los procedimientos para tratar el autismo han obtenido cambios importantes en el entorno educativo global, ya que la educación ha sido el procedimiento principal que se ha caracterizado por producir cambios importantes que normalizan las experiencias de las personas autistas, aunque no "sanen" el autismo. La sintomatología del autismo 
ha ido disminuyendo gracias al manejo de diferentes metodologías manejadas por ciertas instituciones, las cuales producen cambios importantes en la calidad de vida de la persona con este trastorno, puesto que se empieza a manejar desde la niñez y, así, su desarrollo cognitivo va mejorando cada vez más. (p.15)

El sistema educativo de la ciudad de Popayán, aún no ha logrado abarcar con gran éxito a los niños y niñas con autismo, debido a la falta de material didáctico. Esto conlleva a que estas personas no tengan un buen desarrollo en su vida cotidiana. Muchos de los materiales existentes son realizados por los propios profesionales en psicología, los cuales son apropiados debido a la metodología que contienen, pero es necesario aumentar el apoyo visual y estratégico. Actualmente, en esta Ciudad, existen lugares dispuestos para ayudar tanto a adultos como a niños y niñas con autismo. En este caso, se habla del Centro de Especialistas Neurokids, el cual es una institución de rehabilitación infantil para infantes con diferentes trastornos. Se decidió trabajar el proyecto en este centro, ya que él carece de material didáctico para el apoyo en terapias; además, no se ha logrado manejar una metodología permanente que ayude a la educación y en sus terapias.

De esta manera, el proyecto se basa en dar un apoyo en la parte de la metodología de la educación del Centro Neurokids, centrándose en niños y niñas de 6 a 10 años, abordando temas de fauna colombiana, resaltar algunos animales representativos del país y del Departamento del Cauca, constituyéndose en un proyecto adecuado para que dichos niños y niñas logren captar y conocer a la fauna de Colombia, además de reforzar temas como geografía, números, ortografía, asociar colores y el abecedario.

El material ayuda a solucionar una de las principales dificultades de los niños y niñas con esta patología, que consiste en la dificultad para relacionarse con su entorno y asumir el aprendizaje. Propone, inicialmente, nuevas estrategias al Centro Neurokids, con el fin de desarrollar otras herramientas para la inclusión de las personas con este trastorno en el sistema educativo.

De igual forma, no solamente se busca mejorar en el entorno educativo sino, también, en el entorno personal, ya que lo esencial es llegar a que los niños y niñas logren una vida plena.

\section{Metodología}

Para el desarrollo del proyecto, se planteó el proceso de design thinking de Tim Brown; este proceso permite partir de las necesidades del público objetivo, con la intención de aprender, primero, sobre este público, qué necesita y cómo se comporta, para este caso los niños y niñas autistas. La metodología giró alrededor de la experiencia de ellos y permite identificarse con este público. Se consideraron siete etapas no lineales: comprender, observar, definir, idear, prototipar, testear e implementar (MacGuffin, 2018). 
Igualmente, se trabajó con el mapa de empatía, ya que es una herramienta para conocer a la población objetivo, para tener presente cómo piensa, qué siente qué ve, que dice o hace el niño y la niña.

Es importante resaltar, que cada uno de los procedimientos, es importante desarrollar, pues se necesita conocer a fondo a este tipo de población, para lograr idear un material preciso que supla la necesidad encontrada, además de estudiar autores que tratan la parte de psicología y neuropsicología del autismo, para integrarlos con el diseño.

Las técnicas que se aplicaron fueron:

Entrevista: semiestructurada que permite que se expresen más abiertamente en las respuestas y dirigida a los padres de familia con la finalidad de conocer en profundidad lo relacionado a los niños y niñas con los que se trabajó.

Grupo focal: esta se planteó para obtener información con una participación activa de los niños y niñas con autismo, ya que es efectiva para identificar sus necesidades.

Observación no participante: el objetivo de esta herramienta, es hacer un buen análisis del grupo con el que se trabaja, sus actitudes, comportamientos y otros aspectos.

\section{Resultados}

Se realizó un trabajo conjunto con algunos especialistas, para desarrollar una lluvia de ideas de posibles soluciones para la problemática y, así mismo, se desarrolló paso a paso el proceso del design thinking, para la creación de la idea final. Se decidió adaptar a la idea final, diferentes temáticas a las necesidades educativas que tienen los niños y niñas con autismo, con el propósito de promover estrategias de educación que los impulse al aprendizaje de varias ciencias, como las matemáticas, biología, geografía, entre otras, puesto que es el único material existente en este lugar, por lo que es necesario que se tenga variedad de temas y mecanismos.

Luego se realizó un prototipo, el cual tiene como pieza principal un mazo de 28 cartas (véase figura 1), cada una posee una letra del abecedario acompañada con una ilustración de un animal de la fauna del país, con su nombre, especie y localidad donde se ubica, con lo cual el juego da a conocer toda la fauna colombiana, además de apoyar al niño y niña en diferentes temáticas educativas. El prototipo también contiene un rompecabezas, un libro para colorear, stickers de colección, colores, manual de instrucciones, borrador y sacapuntas; cada tipo de pieza mencionada, tiene su objetivo propio, como potencializar habilidades propias de este tipo de niños y, así, reforzar las diferentes funciones y actividades cerebrales. 


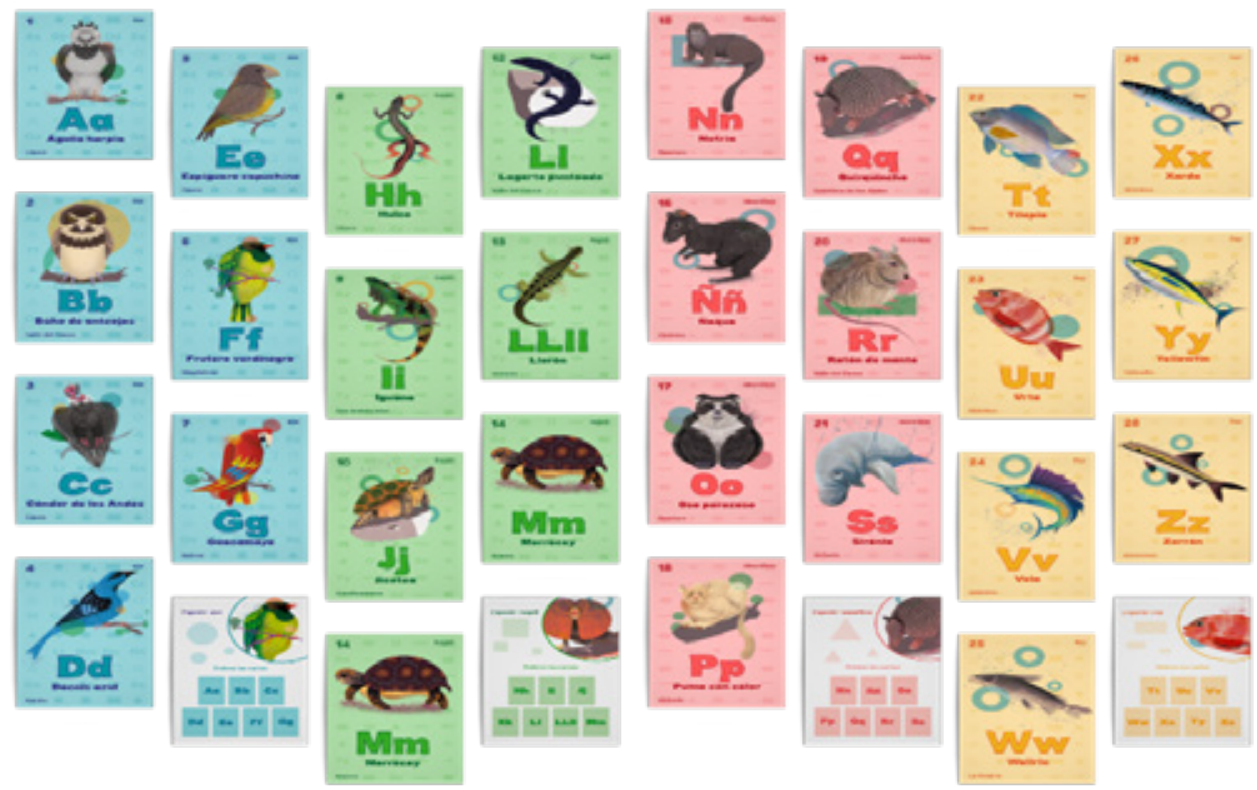

Figura 1. Mazo de 28 cartas Fuente: elaboración propia

Una de las razones por la cual se crea un prototipo físico y no virtual (App), es disminuir el aislamiento del niño y la niña, puesto que, según estudios de profesionales en psicología, los medios virtuales causan manifestaciones tanto visuales como cerebrales que afectan el comportamiento del niño, es decir, este tipo de medios influye en la forma como interactúa el niño o la niña con la sociedad y evita la inmersión en el mundo real (García, 2014).

Asimismo, este tipo de material favorece el desarrollo cognitivo, memorización, conocimiento de su entorno a nivel nacional, al promover, de igual manera, el fortalecimiento de la comunicación e interacción social para lograr superar los síntomas fundamentales del autismo. Así entonces, constituye un material integrado por la psicología y el diseño.

El nombre de este prototipo es Savant, y su slogan es Mi mundo interno (véase figura 2). Desarrolla una modalidad de juego simple pero divertida, para enseñar de forma didáctica las diferentes temáticas que cada especialista en neuropsicología crea pertinente, según las terapias a realizar con ellos y ellas. También le permite al neuropsicólogo(a) decidir qué tipo de actividades quiere realizar con el prototipo, puesto que, como se ha mencionado, es una herramienta integral y flexible. 


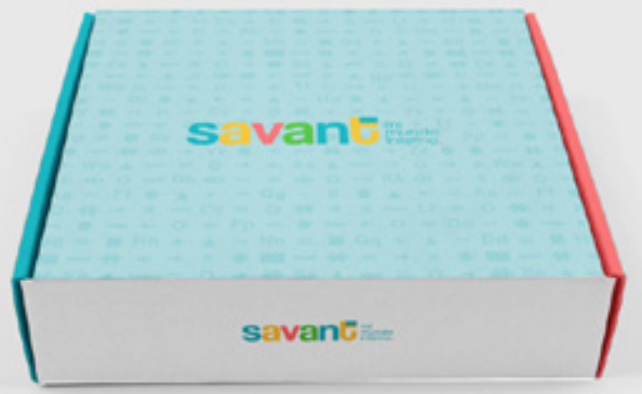

Figura 2. Marca y slogan

Fuente: elaboración propia

\section{Mecánica Principal del Juego}

Para iniciar el juego se necesita que el número de jugadores o jugadoras sean 2 o 4 y se ubiquen uno al lado del otro (véase figura 3). Luego, a cada jugador se le reparte al azar una carta de apoyo para que sepa el grupo de especie del animal, letra y color que tiene que armar; seguido de esto, dependiendo del número de jugadores, se reparte el número de cartas, es decir, si son 2 jugadores, a cada uno(a) le corresponderá 14 cartas, y si son de 4 jugadores, a cada uno(a) le corresponderá 7 cartas. Se tendrá un mazo complementario para que cada jugador pueda tomar cartas y poder armar el grupo que le corresponde. El jugador que primero complete el grupo antes dicho, y por orden alfabético, será el ganador.

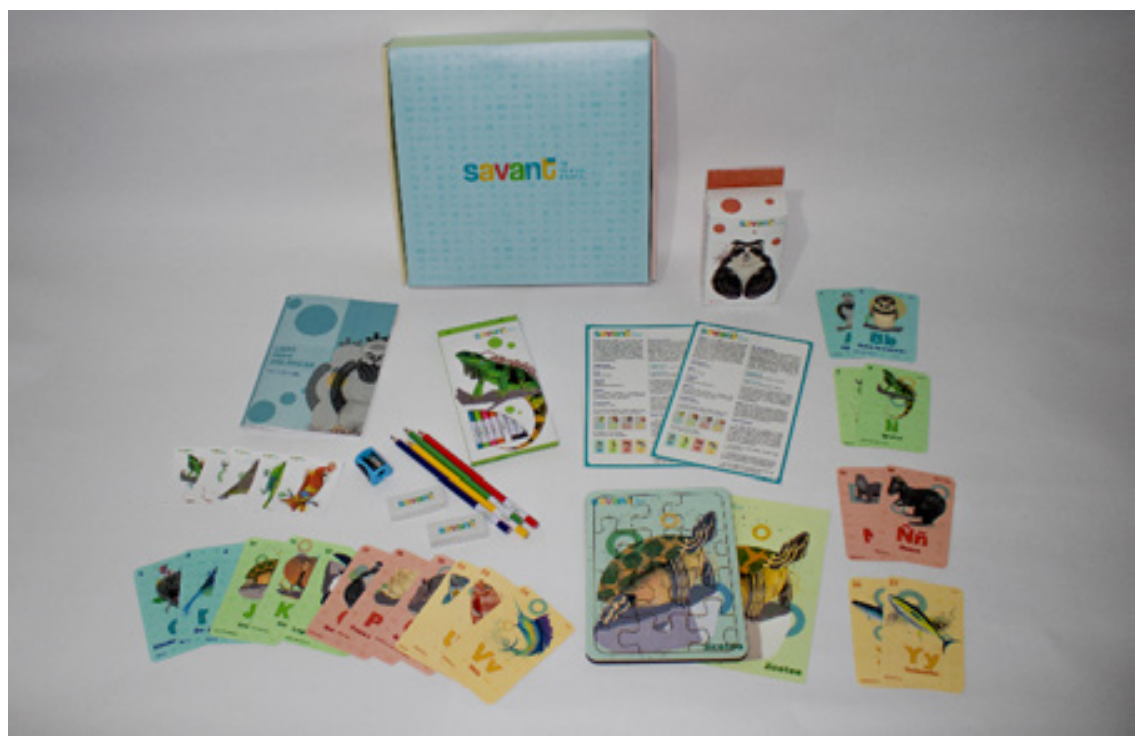

Figura 3. Marca y slogan

Fuente: elaboración propia 
Otro tipo de mecánicas:

- Agrupar por colores

- Agrupar por especies

- Agrupar por localidades

- Ordenar los números de menor a mayor

Reglas de juego:

- En caso de que el jugador(a) tenga alguna carta que no corresponde al color del grupo que debe armar, deberá tomar otra carta y soltar la carta que no corresponde, puesto que solamente se permite tener 7 cartas en caso de 4 jugadores, y 14 cartas en caso de 2 jugadores.

- El jugador(a) sólo puede “comer" (tomar) una carta por cada turno.

- El jugador(a) que complete el grupo que le corresponde, gana si tiene todo su grupo de letras completo y en orden alfabético.

- No se podrá ver de qué color son las cartas del mazo complementario.

Nota: de igual forma, el juego también se puede usar como instructivo para explicar las vocales, las mayúsculas y minúsculas, los fonemas, los números, entre otros, pues esto depende de lo que el especialista le quiera dar a conocer al niño y niña autista.

Finalmente, se demuestra el importante trabajo que tiene el Diseñador Visual en la sociedad, al crear herramientas para mejorar la calidad de vida de las personas, en este caso de los niños y niñas con autismo, dándoles a ellos y ellas un material para llevar a cabo sus habilidades, puesto que es una población que ha sido excluida de la educación. 


\section{Referencias}

García, L. (enero-junio, 2014). El autismo, una condición del pensamiento. Folios de Humanidades y Pedagogía, 1, 27-36. Recuperado de https://revistas.pedagogica. edu.co/index.php/FHP/article/view/2554/2367

Gardner, H. (1983). Inteligencias múltiples. Recuperado en <https://s3.amazonaws.com/ academia.edu.documents/40075791/La_Teoria_de_las_Inteligencias_Multiples_ cortad.pdf?response-content-disposition=inline $\% 3 B \% 20$ filename\%3DLa Teoria_de_las_Inteligencias_Multiples.pdf\&X-Amz-Algorithm=AWS4-HMACSHA256\&X-Amz-Credential=AKIAIWOWYYGZ2Y53UL3A\%2F20190902\%2F us-east-1\%2Fs3\%2Faws4_request\&X-Amz-Date=20190902T235717Z\&X-AmzExpires $=3600 \& X-A m z-S i g n e d H e a d e r s=h o s t \& X-A m z-S i g n a t u r e=c 5 c a d f 691695 f b$ 5b981dd0723b55ff96dc50d40d21d52f427ec9224fffec66b1.

MacGuffin. (2018). Qué es el Design Thinking. Madrid, España. Recuperado de https://www. macguffin.es/blog/qu\%C3\%A9-es-el-design-thinking

Martoz, J. (enero, 2005). Intervención educativa en autismo desde una perspectiva psicológica. Revista de Neurología, 40(1). Recuperado de https://www.neurologia. com/articulo/2005055

Mootee, I. (Ed.). (2014). Design Thinking para la innovación estratégica. Madrid, España: Empresa Activa Editorial.

Riviére, Á. (2001). Orientaciones para la intervención educativa. Madrid: Editorial Fernández Ferraz. 